\title{
Aged Ossabaw swine exhibit intimal medial thickening of coronary arteries similar to that of humans
}

Trevor Lehr, Chris Arnold, John Strobel, James Byrd, Mouhamad Alloosh, and Michael Sturek

Department of Anatomy, Cell Biology, \& Physiology, Indiana University School of Medicine, Indianapolis, IN

Background: Coronary artery disease (CAD) continues to be one of the leading causes of death in the United States. A major contributing factor to CAD is intima media thickening (IMT), which is an early stage in CAD progression to a complex, flow-limiting stenosis. Ossabaw swine have been shown to be exemplary models of human cardiovascular disease, yet there is a paucity of data in animal models of aging. We hypothesized that aged swine would have increased IMT compared to healthy young swine.

Methods: Young (3.1 \pm 0.5 years, $\mathrm{N}=3$ ) and old (10.7 \pm 1.2 years, $\mathrm{N}=3$ ) Ossabaw miniature swine were fed a normal lean diet. Epicardial coronary arteries were interrogated in vivo with IVUS. Images were analyzed every millimeter over the most proximal 45 millimeters of the artery. Intimal thickening (wall coverage; echogenic) was measured as a percentage of total circumference of the vessel lumen. IMT included the echolucent medial layer and was measured as a percentage of total arterial area. Data were represented as mean \pm SEM and $p$ $<0.5$ was deemed significant by independent $t$-test.

Results: Aged swine showed greater IMT compared to the younger swine $(18.7 \pm 1.2 \%$ versus $7.0 \pm 1.3 \% ; p<.002)$. Differences in wall coverage proved not to be significant, as old swine measured $20.3 \pm 4.4 \%$ and young swine measured $14.5+4.8 \%(p<.22)$.

Conclusion: Our data support the hypothesis that old Ossabaw swine exhibited increased intima media thickening compared to lean young swine. This finding further exhibits how coronary artery disease, quantified with clinically used IVUS in the Ossabaw swine model, translates exceptionally well to humans. 